\title{
Study of Three Body $B$ Decays at Belle
}

\section{Paoti Chang* for the Belle Collaboration}

Department of Physics, National Taiwan University, Taipei, Taiwan, R.O.C. E-mail: pchang@phys.ntu.edu.tw!

ABSTRACT: We report preliminary results on 3-body $B$ decays using a data sample of 21.3 $\mathrm{fb}^{-1}$ recorded at the $\Upsilon(4 S)$ resonance with the Belle detector at the KEK $e^{+} e^{-}$storage ring. With no assumption on the intermediate mechanisms, the following branching fractions have been measured for the first time: $\mathcal{B}\left(B^{+} \rightarrow K^{+} \pi^{-} \pi^{+}\right)=(58.5 \pm 7.1 \pm$ $8.8) \times 10^{-6}, \mathcal{B}\left(B^{+} \rightarrow K^{+} K^{-} K^{+}\right)=(37.0 \pm 3.9 \pm 4.4) \times 10^{-6}$ and $\mathcal{B}\left(B^{0} \rightarrow K^{+} \pi^{-} \pi^{0}\right)=$ $\left(35.6_{-7.7}^{+8.1} \pm 5.2\right) \times 10^{-6}$. Results of studies for various intermediate two-body decays are also presented.

During the past few years, a considerable amount of new information on charmless hadronic $B$ decays have been reported [i]. These charmless $B$ decays are particular useful for understanding the dynamics of $B$ meson decays and the origin of $C P$ violation. However, because of large combinatoric backgrounds earlier experimental studies have concentrated mainly on two-body decay processes. In this paper, we report results of a search for $B$ meson decays to $h^{+} h^{-} h^{+}$and $h^{+} h^{-} \pi^{0}(h=K, \pi)$ final states without assuming intermediate hadronic resonances. Inclusion of charge conjugate modes is implied throughout the text. The data sample used in this analysis consists of $21.3 \mathrm{fb}^{-1}$ on the $\Upsilon(4 S)$ resonance and $2.3 \mathrm{fb}^{-1}$ taken $60 \mathrm{MeV}$ below the resonance. The data were collected by the Belle

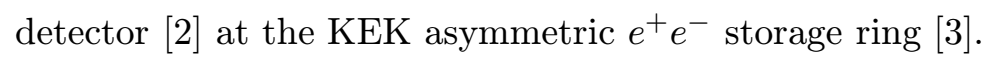

A detailed description of the Belle detector can be found in Ref. [20] $]$ and the event selections of this analysis are described in Ref. [橧]. Charged kaons and pions are identified using a likelihood ratio $L_{K} /\left(L_{\pi}+L_{K}\right)$. Here $L_{K(\pi)}$ is the likelihood of a particle being as a $K(\pi)$ based on $d E / d x$, time of flight and the information from the aerogel Cerenkov counters. $B$ meson candidates are identified using the beam constrained mass $M_{b}=\sqrt{E_{\text {beam }}^{2}-P_{B}^{2}}$ and the energy difference $\Delta E=E_{B}-E_{\text {beam }}$, where $E_{B}$ is the beam energy, and $P_{B}$ and $E_{B}$ are the momentum and energy of a $B$ candidate in the $\Upsilon(4 S)$ rest frame. Events with $M_{b}>5.2 \mathrm{GeV} / c^{2}$ and $|\Delta E|<0.2 \mathrm{GeV}\left(-0.35<\Delta E<0.25\right.$ for $h^{+} h^{-} \pi^{0}$ mode $)$ are selected for the final analysis. The dominant background for this analysis comes from the $e^{+} e^{-} \rightarrow q \bar{q}$ continuum. Various shape variables used to distinguish spherical decay

${ }^{*}$ Speaker. 

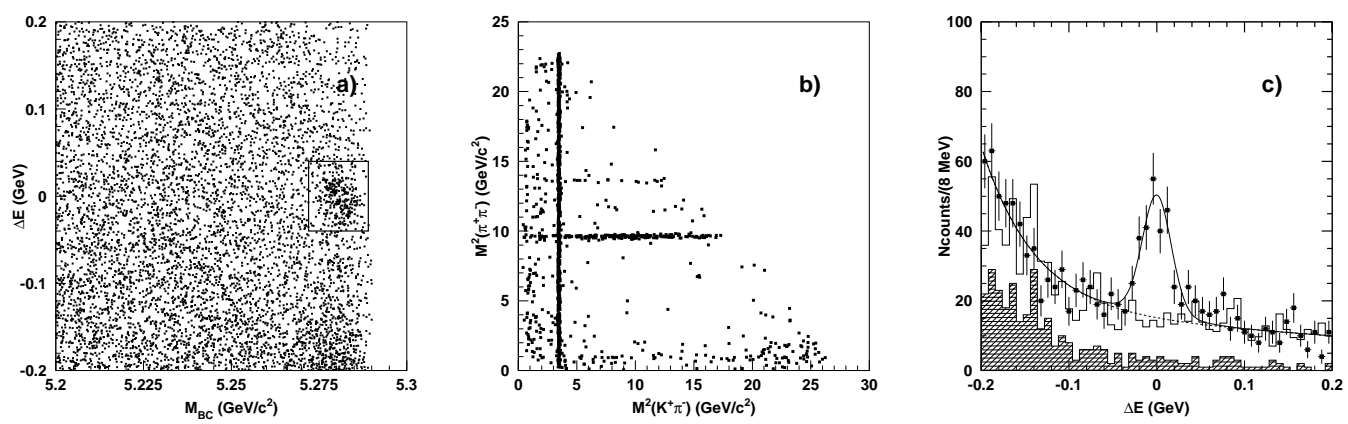

Figure 1: a) $\Delta E$ vs $M_{b}$ distribution for $K^{+} \pi^{-} \pi^{+}$events. b) The Dalitz distributions for events in the $B^{+} \rightarrow K^{+} \pi^{-} \pi^{+}$signal box. c) $\Delta E$ distribution for events in the $M_{b}$ signal box after removing $\bar{D}^{0} \pi^{+}$and $J / \psi(\psi(2 s)) K^{+}$candidates. Points are data, open histograms correspond to the sum of off-resonance data and $B \bar{B}$ Monte Carlo, and hatched histograms are for $B \bar{B}$ Monte Carlo only.

topology ( $B$ events) and jet like characteristics ( $q \bar{q}$ events) are combined to form a Fisher discriminant or likelihood ratio. Continuum suppression is then achieved by applying a cut on the Fisher discriminant or likelihood ratio [4in

Fig. $i_{-i}^{-}$a shows the $\Delta E$ vs $M_{b}$ distribution for $K^{+} \pi^{-} \pi^{+}$events after the continuum background suppression. A clear enhancement can be seen in the signal region. We further investigate those events in the signal region by plotting their Dalitz distributions (see Fig. i: where $J / \psi(\psi(2 s)) \rightarrow \mu^{+} \mu^{-}$are apparent in the Dalitz plot. After removing those candidates with $\bar{D}^{0}$ and $J / \psi(\psi(2 s))$ signals, a clear peak remains as shown in Fig.1.i.c. To investigate possible two-body states in the observed $B^{+} \rightarrow K^{+} \pi^{-} \pi^{+}$signal, we analyze the $K^{+} \pi^{-}$and $\pi^{+} \pi^{-}$invariant mass spectra (see Fig. in the $K^{+} \pi^{-}$spectrum: a narrow peak around $0.9 \mathrm{GeV} / c^{2}$ identified as $K^{* 0}(892)$ and a broad enhancement above $1.0 \mathrm{GeV} / c^{2}$ referred to $K_{x}(1400)$. The $\pi^{+} \pi^{-}$mass spectrum can be described by a narrow resonance $f_{0}(980)$, a broad enhancement between $1.0 \mathrm{GeV} / c^{2}$ to $1.5 \mathrm{GeV} / c^{2}$ which is referred to $f_{x}(1300)$, and a sharp peak around $3.4 \mathrm{GeV} / c^{2}$ that is consistent with $\chi_{c 0}$ meson.

For further analysis, we first exclude the $\chi_{c 0}$ candidates with the requirement that $\left|M\left(\pi^{+} \pi^{-}-3.415\right)\right|>0.05 \mathrm{GeV} / c^{2}$, and then subdivide the Dalitz plot area into seven nonoverlapping regions. The first five regions correspond to $B^{+} \rightarrow K^{* 0} \pi^{+}, B^{+} \rightarrow K_{x}(1400) \pi^{+}$, $B^{+} \rightarrow \rho^{0} K^{+}, B^{+} \rightarrow f_{0}(980) K^{+}$, and $B^{+} \rightarrow f_{x}(1300) K^{+}$final states. The area that $K \pi$ and $\pi \pi$ resonances overlap is covered by region VI and region VII contains the rest of the Dalitz plot. Signal yields in each region is then obtained by $\Delta E$ fits using events in the $M_{b}$ signal region. The $B^{+} \rightarrow K^{+} \pi^{+} \pi^{-}$yield is estimated by summing the signal yields in the seven regions and their reconstruction efficiency is determined from the Monte Carlo simulations of events that are generated with an uniform phase space distribution. To determine the branching fractions for $B$ meson decay to a particular final state $f$, we normalize our results to the observed $B^{+} \rightarrow \bar{D}^{0} \pi^{+}, \overline{D^{0}} \rightarrow K^{+} \pi^{-}$signal using the following 

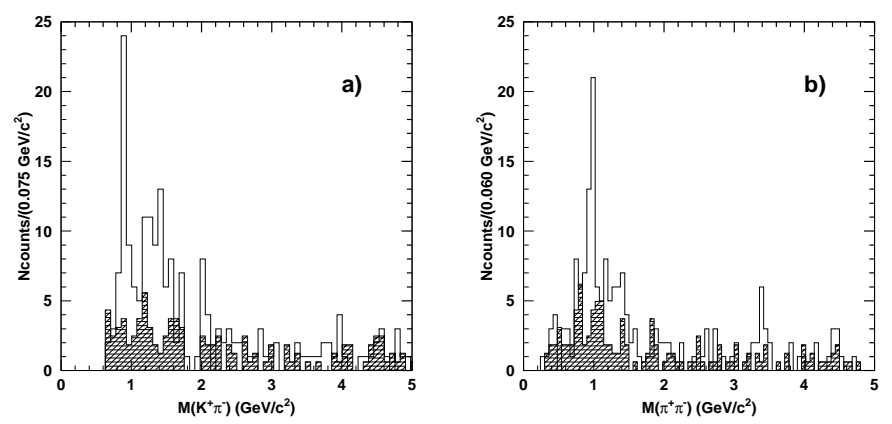

Figure 2: The $K \pi$ and $\pi \pi$ mass spectra for events in the $B^{+} \rightarrow K^{+} \pi^{+} \pi^{-}$signal region (open histograms) and outside the signal region (hatched histograms.

formula:

$$
\mathcal{B}\left(B^{+} \rightarrow f\right)=\mathcal{B}\left(B^{+} \rightarrow \overline{D^{0}} \pi^{+}\right) \times \mathcal{B}\left(\bar{D}^{0} \rightarrow K^{+} \pi^{-}\right) \times \frac{N_{f}}{N_{D \pi}} \times \frac{\varepsilon_{D \pi}}{\varepsilon_{f}},
$$

where $N_{f}$ and $N_{D \pi}$ are number of reconstructed events for states $f$ and $D \pi ; \varepsilon_{f}$ and $\varepsilon_{D \pi}$ are their corresponding efficiencies. The study result of $B^{+} \rightarrow K^{+} \pi^{+} \pi^{-}$decay is summarized in Table 1. In the branching fraction column, the first quoted error is statistical and the second is systematic error that includes the uncertainties due to nonuniformity of the Dalitz distribution, $B^{+} \rightarrow \bar{D}^{0} \pi^{+}$and $\overline{D^{0}} \rightarrow K^{+} \pi^{-}$branching fractions, signal and background shapes in $\Delta E$, and kaon identification efficiency.

To measure the branching fractions of exclusive two-body final states, we need to take into account the possible interference between wide resonances. For this analysis, we consider 5 two-body states in $K^{+} \pi^{+} \pi^{-}$events: $K^{* 0}(892) \pi^{+}, K_{x}(1400) \pi^{+}, \rho^{0}(770) K^{+}, f_{0}(980) K^{+}$, and $f_{x}(1300) K^{+}$. States $K_{x}(1400)$ and $f_{x}(1300)$ are assumed to be $0^{++}$scalars with $\Gamma=294 \mathrm{MeV}$ and $\Gamma=400 \mathrm{MeV}$, respectively. The signal yield in each two-body final state is obtained by a simultaneous fit on $\Delta E$ for the seven regions of the Dalitz plot. The number of signal events, $n_{j}$, in region $j$ of the Dalitz plot can be expressed as

$$
n_{j}=\sum_{i=1}^{5} \varepsilon_{i j} N_{i},
$$

where $N_{i}$ is the number of signal events in state $i$, and $\varepsilon_{i j}$ is the probability for the $i$-th final state to contribute to the $j$-th region. The $\varepsilon_{i j}$ matrix is determined from a Monte Carlo simulation including the reconstruction efficiency. The fit results and the corresponding branching fractions are given in Table 2. Three types of errors are presented: the first error is statistical, the second is systematic and the third reflects the uncertainty due to the interference between different resonant states. This interference effect is modeled in the Monte Carlo simulation to determine $\varepsilon_{i j}$ by varying the relative phases of the amplitudes of different resonant states. A significant uncertainty introduced by the wide resonances is found to be large. 
Table 1: Summary of $B$ decays to three body final states.

\begin{tabular}{lccc} 
Mode & Yield, event & Efficiency, $\%$ & $\mathcal{B}, 10^{-6}$ \\
\hline$K^{+} \pi^{-} \pi^{+}$ & $177 \pm 20$ & 16.2 & $58.5 \pm 7.1 \pm 8.2$ \\
$K^{+} K^{+} K^{-}$ & $162 \pm 16$ & 24.5 & $37.0 \pm 3.9 \pm 4.4$ \\
$K^{-} \pi^{+} \pi^{+}$ & $3.86_{-7.75}^{+8.23}$ & 16.2 & $<7.7$ \\
$K^{+} K^{+} \pi^{-}$ & $6.78_{-4.17}^{+4.87}$ & 13.3 & $<6.0$ \\
$K^{+} K^{-} \pi^{+}$ & $32.9_{-8.80}^{+9.49}$ & 13.2 & $<21$ \\
$K^{+} \pi^{-} \pi^{0}$ & $105.2_{-22.9}^{+23.9}$ & 12.8 & $35.6_{-7.7}^{+8.1} \pm 5.2$ \\
\hline
\end{tabular}

In the search of $B^{+} \rightarrow K^{+} K^{+} K^{-}$decays, we combine three charged tracks that are positively identified as kaons. After vetoing $B$ candidates with any pair of two tracks that is consistent with $D^{0} \rightarrow K^{-} \pi^{+}$and $D_{C P}^{0} \rightarrow K^{+} K^{-}$hypothesis, a clear enhance-

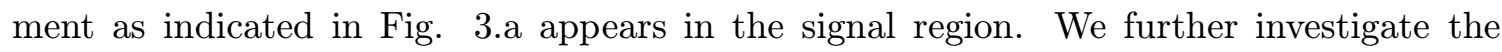
possible two-body resonant states by comparing the $K^{+} K^{-}$invariant mass distribution for events inside and outside the $B$ signal region. Since there are two $K^{+} K^{-}$pairs in each event, we distinguish the $K^{+} K^{-}$combinations with smaller $\left(M\left(K^{+} K^{-}\right)_{\min }\right)$ and the

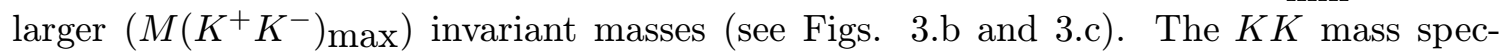
tra can be characterized by a narrow peak at $1.02 \mathrm{GeV} / c^{2}(\phi(1020))$, a broad structure around $1.5 \mathrm{GeV} / c^{2}$, referred as $f_{x}(1500)$, and a peak around $3.4 \mathrm{GeV} / c^{2}$ considered as $\chi_{c 0}$ meson. We first remove the $\chi_{c 0} K^{+}$candidates and then subdivide the Dalitz plot $\left(M^{2}\left(K^{+} K^{-}\right)_{\min }\right.$ vs $\left.M^{2}\left(K^{+} K^{-}\right) \max \right)$ into four non-overlapping regions. The first two correspond to $B^{+} \rightarrow \phi(1020) K^{+}$and $B^{+} \rightarrow f_{x}(1500) K^{+}$, and regions III and IV cover the rest part of the Dalitz plot. The same strategy used in the $K^{+} \pi^{+} \pi^{-}$analysis is applied to extract the $B$ signal yields. The fit results are given in Tables 1 and 2 .

Since a clear $B^{+} \rightarrow \chi_{c 0} K^{+}$signal is seen in both $K^{+} \pi^{+} \pi^{-}$and $K^{+} K^{+} K^{-}$channels, we extract signal yields by fitting the $\pi \pi$ and $K K$ mass spectra of events in the signal region. The fit results are shown in Table 2 and the third error of the branching fraction
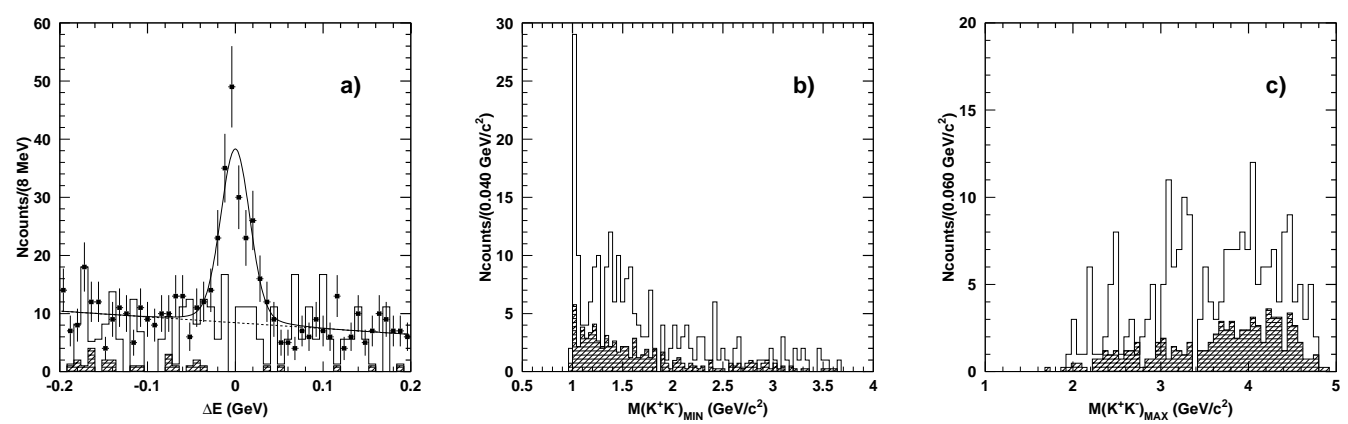

Figure 3: a) The $\Delta E(\mathrm{a}))$ and $K K(\mathrm{~b})$ and c)) invariant mass distributions for $K^{+} K^{+} K^{-}$candidates. 
Table 2: Fit results of $B$ meson decays to exclusive two-body final states.

\begin{tabular}{lccccc} 
Mode & Eff. & Yield & Sig. & $\mathcal{B}_{B \rightarrow R h} \times \mathcal{B}_{R \rightarrow h h}$ & $\mathcal{B}_{B \rightarrow R h}$ \\
& $\%$ & & $\sigma$ & $10^{-6}$ & $10^{-6}$ \\
\hline$K^{* 0}(892) \pi^{+}$ & 19.1 & $38.5_{-7.90}^{+8.50}$ & 6.2 & $11.1_{-2.3-1.4-3.9}^{+2.5+1.4+2.0}$ & $16.7_{-3.4-2.1-5.9}^{+3.7+2.1+3.0}$ \\
$K_{X}(1400) \pi^{+}$ & 17.0 & $39.1_{-10.5}^{+10.8}$ & 4.1 & $12.2_{-3.3-1.8-5.8}^{+3.4+1.7+9}$ & - \\
$\rho^{0}(770) K^{+}$ & 18.9 & $1.75_{-7.38}^{+8.60}$ & 0.2 & $<9.6$ & $<9.6$ \\
$f_{0}(980) K^{+}$ & 19.3 & $40.9_{-9.56}^{+8.85}$ & 6.0 & $11.7_{-2.7-1.5-1.1 .0}^{+2.5+1.5+4.1}$ & - \\
$f_{X}(1300) K^{+}$ & 17.3 & $33.6_{-11.1}^{+12.1}$ & 3.2 & $10.7_{-3.5-1.4-2.8}^{+3.9+1.4 .9}$ & - \\
$\phi(1020) K^{+}$ & 25.1 & $30.1_{-6.52}^{+7.35}$ & 6.4 & $6.70_{-1.5}^{+1.6} \pm 0.8 \pm 0.4$ & $13.6_{-3.1}^{+3.3} \pm 1.6 \pm 0.8$ \\
$f_{X}(1500) K^{+}$ & 22.1 & $122_{-14.5}^{+15.1}$ & 12.1 & $30.8_{-3.7-3.9-1.5}^{+3.8+3.9+1.5}$ & - \\
$\chi_{c 0}\left(\pi^{+} \pi^{-}\right) K^{+}$ & 21.5 & $15.5_{-4.6}^{+5.3}$ & 4.8 & - & $8.0_{-2.4}^{+2.8} \pm 1.0 \pm 1.1$ \\
$\chi_{c 0}\left(K^{+} K^{-}\right) K^{+}$ & 13.7 & $7.7_{-3.1}^{+3.9}$ & 3.2 & - & $5.3_{-2.2}^{+2.7} \pm 0.7 \pm 0.8$ \\
$K^{* 0}(892) \pi^{0}$ & 8.7 & $5.5_{-3.5}^{+4.2}$ & 1.7 & $<6.4$ & $<9.6$ \\
$K^{*-}(892) \pi^{+}$ & 6.6 & $13.1 \pm 4.2$ & 4.3 & $8.7 \pm 2.8 \pm 1.2$ & $26.0 \pm 8.3 \pm 3.5$ \\
$\rho^{+} K^{-}$ & 6.0 & $21.9_{-6.4}^{+7.1}$ & 4.2 & $15.8_{-4.6-3.0}^{+5.1+1.7}$ & $15.8_{-4.6-3.0}^{+5.1+1.7}$ \\
$\rho^{0} \pi^{0}$ & 9.0 & $0.0_{-0.0}^{+1.8}$ & - & $<2.8$ & $<2.8$ \\
$\rho^{0} \pi^{+}$ & 11.9 & $15.5_{-11.0}^{+11.8}$ & 1.9 & $<14.5$ & $<14.5$ \\
\hline
\end{tabular}

corresponds to the uncertainty of the $\chi_{c 0} \rightarrow \pi \pi, K K$ decay rates. We also perform $B^{+} \rightarrow$ $K^{-} \pi^{+} \pi^{+}, B^{+} \rightarrow K^{+} K^{+} \pi^{-}$and $B^{+} \rightarrow K^{+} K^{-} \pi^{+}$searches; no significant signals have been seen. Therefore, upper limits at $90 \%$ confidence level are given.

For the events with $K^{+} \pi^{-} \pi^{0}$ in the final state, we find a sizeable contribution from $B \rightarrow \bar{D}^{0} \pi$ decays. After vetoing events that fall in $D^{0}$ mass window, clear peaks appear in both $M_{b}$ and $\Delta E$ spectra as shown in the top two plots to the right of Fig. 'i- Results of $B^{0} \rightarrow K^{+} \pi^{-} \pi^{0}$ is obtained using a $\Delta E$ fit. The plot to the left of Fig. 歪 shows the invariant masses of $K^{+} \pi^{-}, K^{+} \pi^{0}$, and $\pi^{+} \pi^{0}$ using events in the $B$ signal region (open histogram) and $M_{b}$ sideband events (shaded area) defined as $5.2<M_{b}<5.26 \mathrm{GeV} / c^{2}$. Potential candidates of the intermediate states include $K^{* 0}(892) \pi^{0}, K^{*+}(892) \pi^{-}$, and $\rho^{+} K^{-}$(vectorpseudoscalar). An enhancement in the $K^{+} \pi^{-}$spectrum peaking around $1.3 \mathrm{GeV} / c^{2}$ is also observed. We also search for $B^{0} \rightarrow \rho^{0} \pi^{0}$ signals by requiring the $\pi^{+} \pi^{-}$mass to be within $170 \mathrm{MeV} / c^{2}$ of the nominal $\rho$ mass. To prevent cross talk or interference from other decay channels, we require the invariant mass of a vector's daughter and the prompt hadron to be

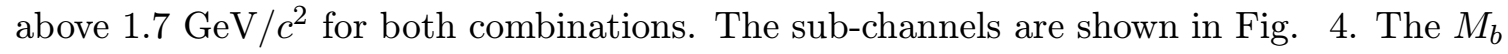
and $\Delta E$ distributions in each sample are fitted using a two dimensional extended unbinned likelihood fit. Statistically, besides the inclusive decay of $B^{0} \rightarrow K^{+} \pi^{-} \pi^{0}$, significant signals in the charmless pseudoscalar-vector modes $B^{0} \rightarrow K^{*+} \pi^{-}$and $B^{0} \rightarrow \rho^{-} K^{+}$are seen. However, the large $K^{+} \pi^{-} \pi^{0}$ signals cannot be explained as a sum of $K^{*+} \pi^{-}$and $\rho^{-} K^{+}$ modes. The enhancement around $1.3 \mathrm{GeV} / c^{2}$ in $K^{+} \pi^{-}$mass contributes a significant amount of $B^{0} \rightarrow K^{+} \pi^{-} \pi^{0}$ decays. At last, we perform a search for $B^{+} \rightarrow \rho^{0} \pi^{+}$decay by fitting $\Delta E$ for the $\pi^{+} \pi^{+} \pi^{-}$candidates with $\pi^{+} \pi^{-}$mass within $150 \mathrm{MeV} / c^{2}$ of the nominal $\rho$ mass. Although there are some signal candidates, the obtained yield is not significant 

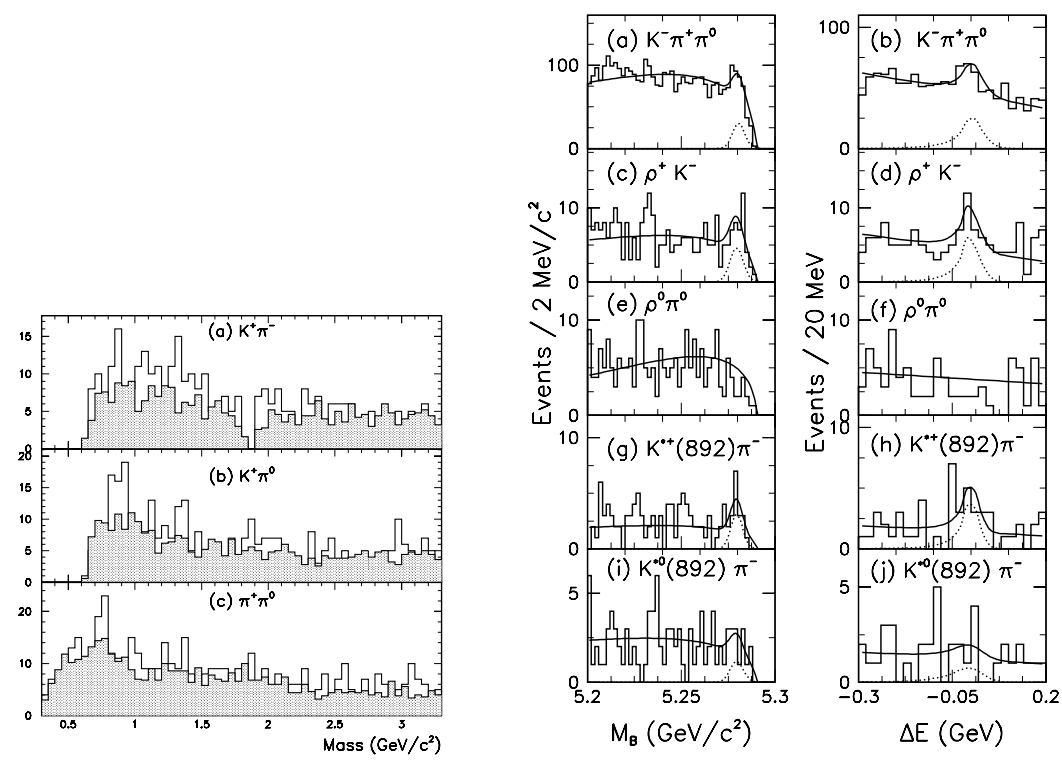

Figure 4: (Left) The $K^{+} \pi^{-}, K^{+} \pi^{0}$ and $\pi^{+} \pi^{0}$ mass distribution for $K^{+} \pi^{-} \pi^{0}$ events in the $B$ signal region. (Right) $M_{b}$ and $\Delta E$ distributions for $K^{+} \pi^{-} \pi^{0}, \rho^{+} K^{-}, \rho^{0} \pi^{0}, K^{*+}(892) \pi^{-}$, and $K^{* 0}(892) \pi^{0}$ candidates. Events in $M_{b}(\Delta E)$ plots are required to be in $\Delta E\left(M_{b}\right)$ signal region after all selection criteria are applied.

and an upper limit is given. Table 2 summarizes the fit results of all sub-channels.

In summary we report the first observation of a large branching fraction for the inclusive three body decays $\mathcal{B}\left(B^{+} \rightarrow K^{+} \pi^{+} \pi^{-}\right)=(58.5 \pm 7.1 \pm 8.8) \times 10^{-6}, \mathcal{B}\left(B^{+} \rightarrow K^{+} K^{+} K^{-}\right)=$ $(37.0 \pm 3.9 \pm 4.4) \times 10^{-6}$ and $\mathcal{B}\left(B^{0} \rightarrow K^{+} \pi^{-} \pi^{0}\right)=\left(35.6_{-7.7}^{+8.1} \pm 5.2\right) \times 10^{-6}$. Earlier measurements on non-resonant three-body decays, $K^{+} \pi^{+} \pi^{-}$(CLEO) and $K^{+} K^{+} K^{-}$(BaBar) only reported upper limits and some of them were lower than our central values. A comparison of selection criteria shows that CLEO and BaBar required the invariant masses of any two particles above $2.0 \mathrm{GeV} / c^{2}$. This requirement effectively removes most of the low mass resonances that provide a dominant contribution to our observed signals. As for other three-body decay modes, $K^{-} \pi^{+} \pi^{+}, K^{+} K^{-} \pi^{+}$and $K^{+} K^{+} \pi^{-}$. stringent upper limits are given. In search of intermediate two-body states, we have observed for the first time in $B$ decays to $\chi_{c 0} K^{+}, K^{* 0}(892) \pi^{+}, K^{*+}(892) \pi^{-}, \rho^{-} K^{+}$, and $f_{0}(980) K^{+}$. The latter is of interest since this is the first observation of a $B$ decay to a charmless scalar-pseudoscalar final state. Two-body decays with high mass resonances, $K_{x}(1400) \pi^{+}, K_{x}(1400) \pi^{0}, f_{x}(1300) K^{+}$, and $f_{x}(1500) K^{+}$have been seen. An angular analysis that requires data with large statistics is needed to identify those resonances. At last we find that the interference effect between different two-body intermediate states could be large. A full amplitude analysis is possible in the future to understand the dynamics of the interference. 


\section{References}

[1] CLEO Collaboration, G. Godang et al., Phys. Rev. Lett. 80, 3456 (1998); CLEO Collaboration, C.P. Jessop et al., Phys. Rev. Lett. 85, 2881 (2000); CLEO Collaboration, D. Cronin-Hennessy et al., Phys. Rev. Lett. 85, 515 (2000); Belle Collaboration, K. Abe. et al., Phys. Rev. Lett. 87, 101801 (2001); Belle Collaboration, K. Abe. et al., Phys. Lett. B, 517, 309 (2001); BaBar Collaboration, B. Aubert et al., Phys. Rev. Lett. 87, 151802 (2001).

[2] Belle Collaboration, K. Abashian et al., KEK Progress Report 2000-4 (2000), to be published in Nucl. Inst. and Meth. A.

[3] KEKB B Factory Design Report, KEK Report 95-7 (1995), unpublished; Y. Funakoshi et al., Proc. 2000 European Particle Accelerator Conference, Vienna (2000).

[4] Belle Collaboration, K. Abe et al., hep-ex/0107051;

Belle Collaboration, K. Abe et al., Contributed paper 946 of XX Int. Symp. on Lepton and Photon Interactions at High Energies, Rome, Italy (2001).

[5] CLEO collaboration, T. Bergfeld et al., Phys. Rev. lett. 77, 4503 (1996);

BaBar collaboration, T.J. Champion, Proc. of the XXXth Int. Conf. on High Energy Phys., Osaka (2000). 